\title{
Photoluminescence Spectroscopy of Carbon Nanotube Bundles: Evidence for Exciton Energy Transfer
}

\author{
P. H. Tan, A. G. Rozhin, T. Hasan, P. Hu, V. Scardaci, W. I. Milne, and A. C. Ferrari* \\ Department of Engineering, University of Cambridge, Cambridge CB3 OFA, United Kingdom
}

(Received 1 February 2007; published 27 September 2007)

\begin{abstract}
We investigate photoluminescence of nanotube bundles. Their spectra are explained by exciton energy transfer between adjacent tubes, whereby excitation of large gap tubes induces emission from smaller gap ones. The consequent relaxation rate is faster than nonradiative recombination, leading to enhanced photoluminescence of acceptor tubes.
\end{abstract}

DOI: 10.1103/PhysRevLett.99.137402

PACS numbers: 78.67.Ch, 71.35.Cc, 78.55.-m

Single wall carbon nanotubes (SWNTs) are rolled graphene sheets [1]. Quantum confinement makes their band structure different from graphene, with subbands and singularities in the density of states [1], fully determined by the chiral indices $(n, m)$. Measuring the optical transitions allows one in principle to determine the chiral indices. Thus, a massive effort was put to measure SWNT photoluminescence (PL) since their discovery. However, it took more than ten years to unambiguously detect and identify PL emission from SWNTs [2,3], once debundling was achieved [2,3]. The discrepancy between single-particle theory and experiments pointed to the major role of electron-electron and electron-hole interactions in shaping their band structure [4,5]. The exciton binding energies were recently measured [4,5]. These are very large, from tens of $\mathrm{meV}$ to $1 \mathrm{eV}$ [4-6]. Thus, excitons dominate even at room temperature.

The investigation of SWNT optical properties is now a highly pursued research area [2-9]; however, this still focuses on individual tubes, in contrast with their tendency to bundle. Furthermore, the PL quantum yield of individual SWNTs is very low and this hinders their applications in optoelectronics $[3,8,9]$. PL excitation (PLE) in bundles was recently studied and assigned to intertube carrier transfer [10]. Here we investigate absorption and emission of SWNT bundles. We show that their apparently complex spectra can be interpreted considering exciton energy transfer (EET) between tubes. This is a well-known phenomenon in biological systems, conjugated polymers, quantum wires, and dots [11-15], which we now identify in SWNTs. We find that it is a major nonradiative relaxation channel, strongly enhancing PL of acceptor tubes. Thus, contrary to what is usually assumed, bundles could be ideal for high yield optoelectronics, surpassing the poor performance of individual tubes [8,9]. Furthermore, EET fingerprints bundles with different semiconducting concentration, offering a means to monitor the composition of bundles in solution, which is key for research and applications $[2,3]$.

We measure absorption on CoMoCAT SWNTs [16] suspensions in $\mathrm{D}_{2} \mathrm{O}$ with sodium dodecylbenzene sulfonate
(SDBS) surfactant [2], using a Perkin-Elmer 950 spectrometer. A JY Fluorolog-3 is used for PLE.

Figure 1(a) plots PLE maps from the as-prepared solution. Each spot can be labeled as $\left(\lambda_{\mathrm{ex}}, \lambda_{\mathrm{em}}\right)$, where $\lambda_{\mathrm{ex}}, \lambda_{\mathrm{em}}$ are, respectively, the excitation and emission wavelengths. Several high intensity peaks are exciton-exciton resonances $[3,17]$. In this case $\lambda_{\text {ex }}$ corresponds to the energy of the excitonic states $e h_{i i}$ associated with the $i$ th electronic interband transitions $E_{i i}(i=1,2,3,4)$ in the singleparticle picture $[3,17]$, while $\lambda_{\mathrm{em}}$ is the emission energy of the lowest exciton transition $e h_{11}$. Other spots in Fig. 1(a) are related to exciton-phonon sidebands [18-20]. The spectral features in Fig. 1(a) are summarized in Fig. 2. Sixteen SWNT species are seen in the range 800-1300 nm. Their chiral indices are assigned in Fig. 2 [3]. The phonon sidebands for the $e h_{11}$ and $e h_{22}$ excitons are shown in

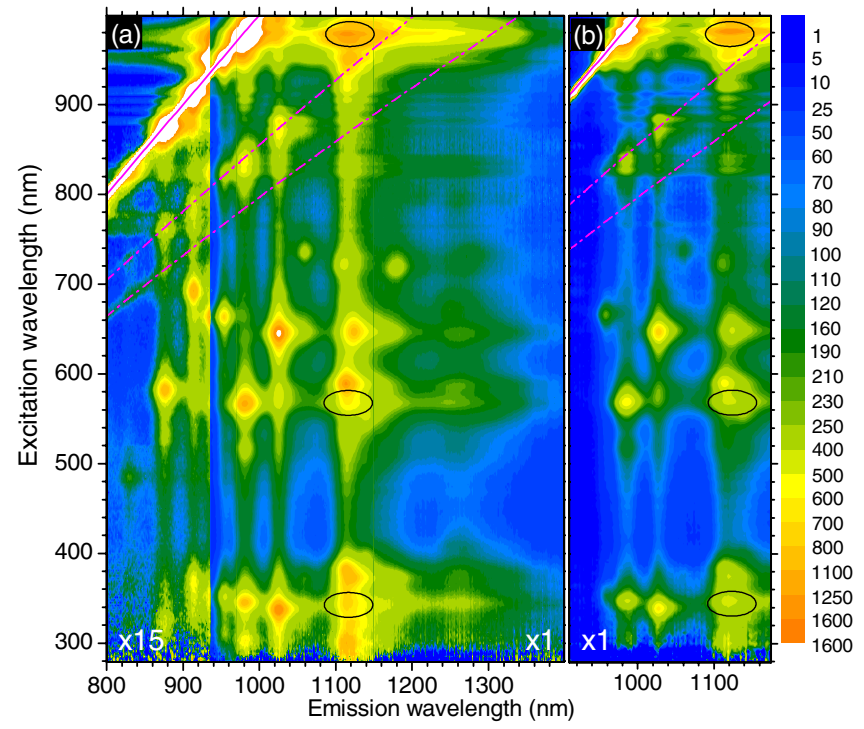

FIG. 1 (color online). PLE map for (a) as-prepared suspensions and (b) after two months. Solid lines at upper left corners represent resonances with same excitation and recombination energies. The dash-dotted lines represent the range of phonon sidebands. Ellipses mark emission from $(8,4),(7,6)$, and $(9,4)$ SWNTs, with excitation matching $e h_{11}, e h_{22}, e h_{33}$ of $(6,5)$. 


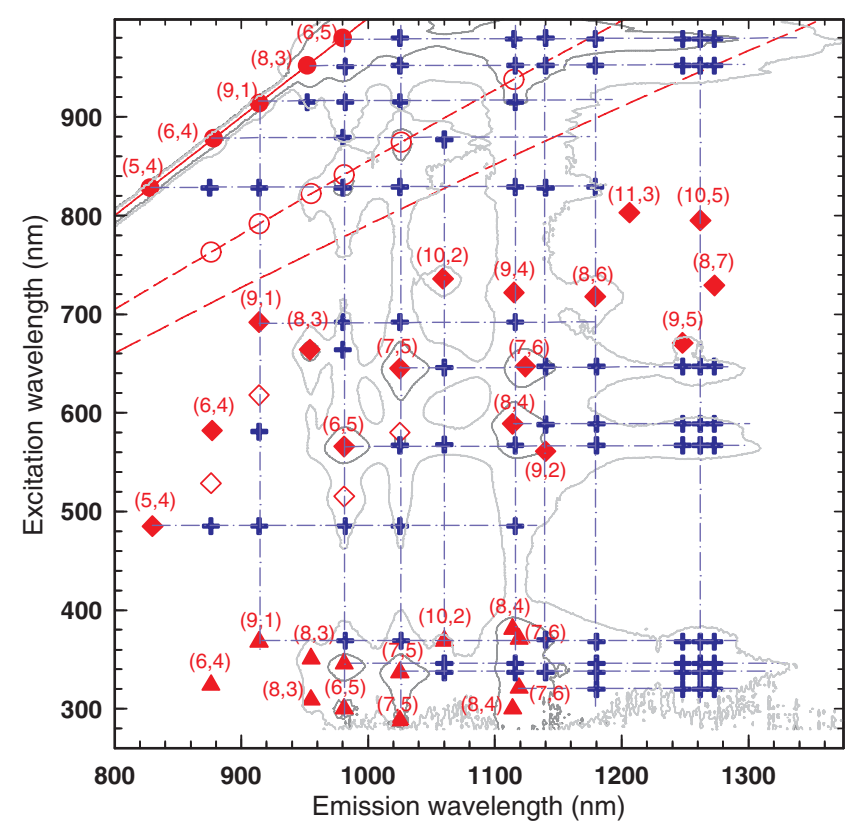

FIG. 2 (color online). PLE of as-prepared suspension. Solid circles, diamonds, and triangles represent $e h_{11}$ emission of SWNTs with excitation matching their $e h_{11}, e h_{22}, e h_{33}, e h_{44}$ transitions. Each peak is labeled with the SWNT chiral index. Open circles and diamonds are phonon sidebands. Solid crosses are EET between $s$-SWNTs. Gray contour patterns comprise both exciton-related resonances and EET spectral features.

Fig. 2 with open circles and diamonds. The $e h_{i i}$ wavelengths of most SWNTs here are 3-10 nm larger than Ref. [3]. This redshift is expected in the presence of bundling [21,22]. Figure 2 has some interesting features compared with previous data on isolated SWNT suspensions [3,17]: (i) the spectral profiles of exciton resonances significantly elongate in the horizontal and vertical directions; (ii) new peaks appear, such as, e.g., $(645 \mathrm{~nm}, 1265 \mathrm{~nm})$ and $(568 \mathrm{~nm}, 1250 \mathrm{~nm})$, with intensity much stronger than the $\left(e h_{22}, e h_{11}\right)$ peaks of $(10,5),(8,7)$, and $(9,5)$ SWNTs; (iii) a strong broad band near $(980 \mathrm{~nm}, 1118 \mathrm{~nm})$ is observed.

To clarify the origin of these bands, we checked PLE from the same solution after two months [Fig. 1(b)]. Figures 1(a) and 1(b) have similar features. However, the $e h_{11}$ emissions of most SWNTs in Fig. 1(b) redshift $\sim 3-5 \mathrm{~nm}$ relative to Fig. 1(a). This suggests further aggregation into bigger bundles, as confirmed by redshift and broadening of the corresponding absorption peaks (see Ref. [23]). Also, almost all peak intensities decrease. But a careful examination of Figs. 1(a) and 1(b) shows that the $(980 \mathrm{~nm}, 1118 \mathrm{~nm})$ band becomes stronger after two months. Also, two peaks near $(568 \mathrm{~nm}, 1118 \mathrm{~nm})$, (346 nm, $1118 \mathrm{~nm})$ [ellipses, Figs. 1(a) and 1(b)], are more clear, due to the lower intensity of the $\left(e h_{i i}, e h_{11}\right)$ $(i=2,3,4)$ bands of $(8,4)$ and $(7,6)$ tubes, which shadowed them in the pristine solution. Notably, these three peaks do not correspond to any of the known exciton-exciton resonances of SWNTs in this spectral range $[3,17]$. The $(980 \mathrm{~nm}, 1118 \mathrm{~nm})$ peak is not assigned to a phonon sideband of $(8,4),(7,6)$, or $(9,4)$ tubes, due to its much lower position than previous investigations of these tubes $[18,19,24,25]$. Indeed, the excitation energies of the $(980 \mathrm{~nm}, 1118 \mathrm{~nm}),(568 \mathrm{~nm}, 1118 \mathrm{~nm})$, and (346 nm, $1118 \mathrm{~nm}$ ) bands match, respectively, the $e h_{11}$, $e h_{22}$, and $e h_{33}$ transitions of $(6,5)$ tubes [3], whereas their emission around $1118 \mathrm{~nm}$ is consistent with $(8,4),(7,6)$, $(9,4) e h_{11}$. Thus, resonant excitation of large gap donors induces emission from smaller gap acceptors. This implies energy transfer between SWNT in bundles. Because of the large exciton binding energies [4-6], this happens via excitons, not intertube electron or hole migration [10].

A thorough examination of all peaks in Figs. 1(a) and 1(b), allows identification of other EET features (solid crosses, Fig. 2). Peaks not attributable to known excitonexciton resonances along each horizontal dash-dotted line in Fig. 2 are assigned to $e h_{i i}$ excitation of donor tubes, inducing $e h_{11}$ emission from acceptors. Vice versa, the crosses along each vertical dash-dotted line are $e h_{11}$ emission of acceptors, following EET from $e h_{i i}$ excitation of donors. The broad or elongated patterns of Fig. 1 (gray contours in Fig. 2) contain overlapping peaks from tubes with similar excitation or emission energies. In a bundle, the concentration and distribution of nanotube species will determine the EET-induced intensities. The higher the concentration of semiconducting tubes, the higher the probability of them being adjacent, the higher the chance of EET-induced emission. Thus, the strongest peaks will appear around $e h_{i i}$ transitions of semiconducting tubes with highest concentration, such as $(6,5),(7,5),(8,4)$ in our CoMoCAT solutions [16,17].

Figure 3 compares the absorption of the as-prepared solution with its PLE and PL spectra. The $\left(e h_{i i}, e h_{11}\right)$ peaks are marked by crosses. We assign most of the other bands to EET from donors to acceptors within bundles. The $\left(e h_{11}, e h_{11}\right)$ peak is the strongest among all possible $\left(e h_{i i}\right.$, $\left.e h_{11}\right)$ for a given $(n, m)$, as, e.g., in the $(6,5)$ tube in Fig. 3(b). This is because $e h_{11}$ excitons have higher density of states than $e h_{22}, e h_{33}$ [26]. Thus, more photons are absorbed by $e h_{11}$ states. Then, as for Fig. 3(a), $e h_{11}$ excitation of large gap donors is a more efficient way to enhance emission of smaller gap acceptors than direct $e h_{22}$ and $e h_{33}$ excitation of the acceptors, despite the low quantum efficiency of individual tubes [2].

We now estimate the EET efficiency. Consider the exciton relaxation of two adjacent tubes with different gaps, following resonant $e h_{11}$ excitation of the larger gap tube. The rate equations of the donor-acceptor system can be written as follows:

$$
\begin{gathered}
\partial n_{D} / \partial t=G_{\mathrm{pe}}-n_{D}\left(1 / \tau_{\mathrm{nr} D}+1 / \tau_{r D}\right)-n_{D} / \tau_{\mathrm{DA}}, \\
\partial n_{A} / \partial t=n_{D} / \tau_{\mathrm{DA}}-n_{A}\left(1 / \tau_{\mathrm{nr} A}+1 / \tau_{r A}\right),
\end{gathered}
$$

where $\tau_{\mathrm{DA}}$ is the energy transfer lifetime between donors 


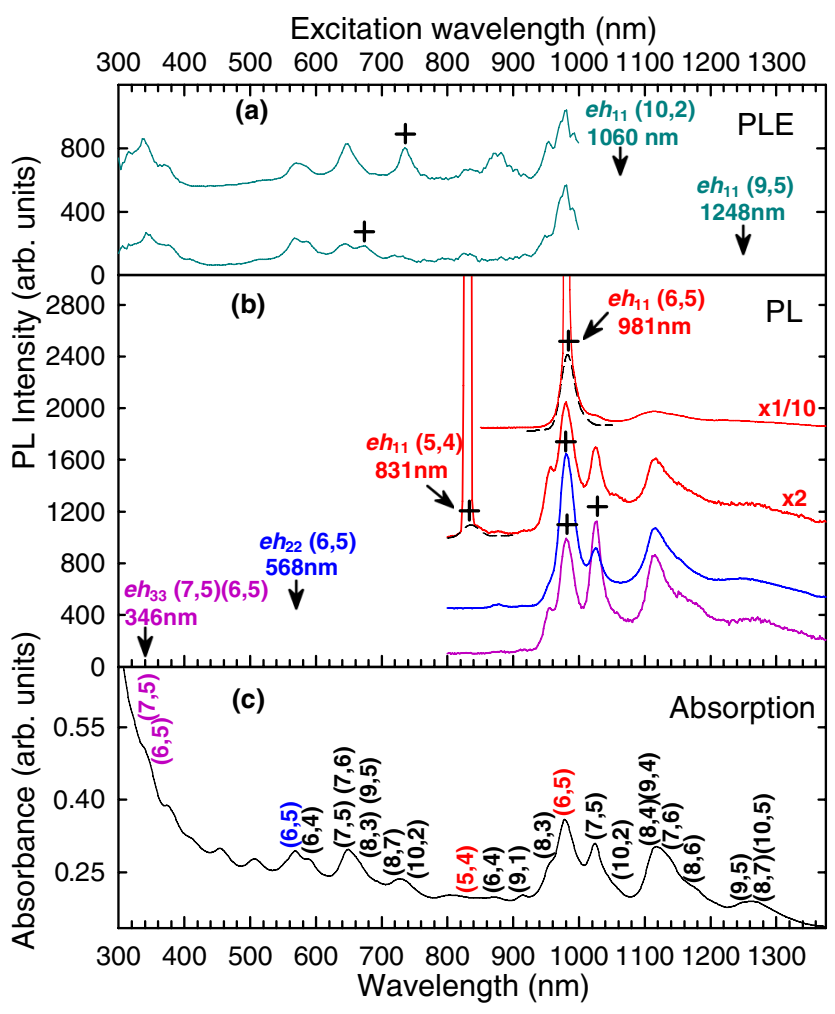

FIG. 3 (color online). (a) PLE, (b) emission, and (c) absorption spectra. The arrows indicate detection wavelengths in PLE and excitation in emission. Crosses in (a),(b) mark exciton-exciton resonances in emission and excitation. Dashed lines in (b) are fits of the $\left(e h_{11}, e h_{11}\right)$ resonances, after subtracting the fitted Rayleigh peaks of the SWNT solution from one without SWNTs.

$(D)$ and acceptors $(A), n_{D}$ is the population of excitons in the donor and $n_{A}$ in the acceptor, $\tau_{\mathrm{nr} D}, \tau_{r D}, \tau_{\mathrm{nr} A}$, and $\tau_{r A}$ are the radiative ( $r$ ) and nonradiative (nr) lifetimes, $G_{\mathrm{pe}}$ the exciton density in the donor created by photoexcitation. An estimation of the EET efficiency is the ratio of acceptor $e h_{11}$ emission intensity $\left(I_{A}=n_{A} / \tau_{r A}\right)$ to that of the donor $\left(I_{D}=n_{D} / \tau_{r D}\right)$. Then, deriving $n_{A} / n_{D}$ from Eqs. (1) and (2) at steady state, we get the following:

$$
I_{A} / I_{D}=\frac{1 / \tau_{\mathrm{DA}}}{1 / \tau_{r A}+1 / \tau_{\mathrm{nrA}}} \frac{\tau_{r D}}{\tau_{r A}} .
$$

The $e h_{11}$ radiative lifetime is reported to be $\sim 20-180$ ps, dependent on temperature [27]. For tube diameters $\sim 0.75-0.95 \mathrm{~nm}$, it is about $\sim 20-30 \mathrm{ps}$ at room temperature [28]. This is much shorter than the theoretical radiative lifetime ( $\sim 10 \mathrm{~ns})$ [29]. Thus, the observed lifetimes are determined by nonradiative recombinations. Equation (3) can then be simplified as $I_{A} / I_{D} \approx \tau_{\mathrm{nr} A} / \tau_{\mathrm{DA}}$.

We measure a very high $I_{A} / I_{D}$ in bundles. E.g., under $e h_{11}$ excitation of the $(5,4)$ tubes in Fig. 3(b), the ratio of photoluminescence intensity of all acceptor tubes with emission above $900 \mathrm{~nm}$ [such as $(6,5),(7,5),(8,4)$, $(7,6)]$ to that at $\sim 831 \mathrm{~nm}$ of the $(5,4)$ donors is at least $\sim 75$. This indicates that most resonantly excited $(5,4) e h_{11}$ excitons transfer their energy to the acceptors, rather than recombine. Thus, in bundles exciton relaxation via EET is comparable or even faster than nonradiative recombination. This fast relaxation suppresses emission from donors, but it significantly increases the acceptors luminescence. The presence of metallic tubes in bundles strongly quenches the luminescence from semiconducting tubes; however, our results suggest that small bundles entirely formed of semiconducting tubes can be ideal for optoelectronics, such as in light-emitting devices $[8,9]$.

Two-photon excitation is used to derive exciton binding energies [4,5]. Figure 4 summarizes the two-photon map of Ref. [5]. Open circles are two-photon exciton resonances. These are slightly shifted with respect to Ref. [4] due to the presence of small bundles [5]. Below each two-photon band, Ref. [5] reported a set of peaks (solid squares and circles, Fig. 4). Each of these matches the excitation energy of a larger gap tube, as indicated by horizontal dashed lines in Fig. 4. These are analogous to the EET-induced peaks along each horizontal dash-dotted line in Fig. 2. We attribute them to emission of small gap tubes due to EET from larger gap tubes in bundles. We assign the four features in Fig. 4 with $\sim 1390 \mathrm{~nm}$ excitation (solid squares) to EET from $(5,4)$ donors to $(6,4),(9,1),(8,3)$, and $(6,5)$ acceptors. Since two-photon luminescence increases quadratically with excitation power, the energy transfer features show more distinct peaks compared to Fig. 1. This is quite remarkable, since Ref. [5] studied HiPCO SWNT, which have a much higher concentration of metallic tubes with respect to the CoMoCAT samples considered here.

In low-dimensional systems, exciton tunneling, photonexchange, and Förster Resonance Energy Transfer (FRET) are efficient EET mechanisms [11-15,30]. We attribute EET in bundles to FRET. Indeed, tunneling requires coupling of exciton wave functions. Its rate is also very sensitive to the $e h_{11}$ energy difference [30]. The 16 tube species in our experiment have diameters $\sim 0.65-1.05 \mathrm{~nm}, e h_{11}$ $\sim 0.06-0.5 \mathrm{eV}$, and chiral angle variation $\sim 5-26^{\circ}[3,17]$. Therefore, the efficiency should strongly depend on spe-

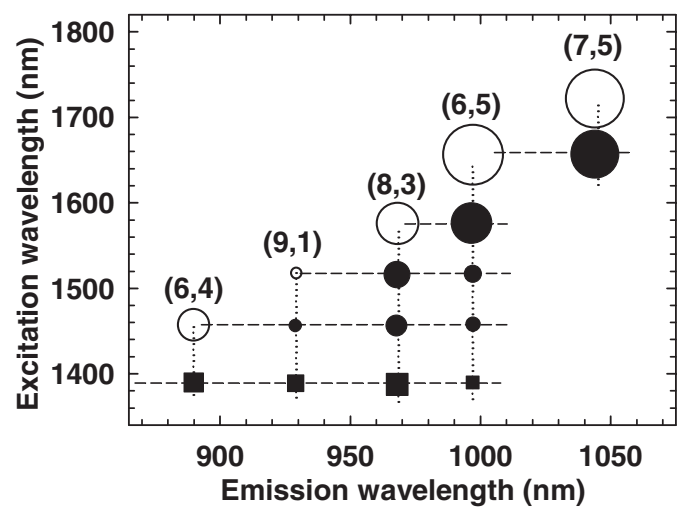

FIG. 4. Schematic representation of the two-photon map of Ref. [5]. Open circles: two-photon peaks. Solid circles, squares: EET peaks. 

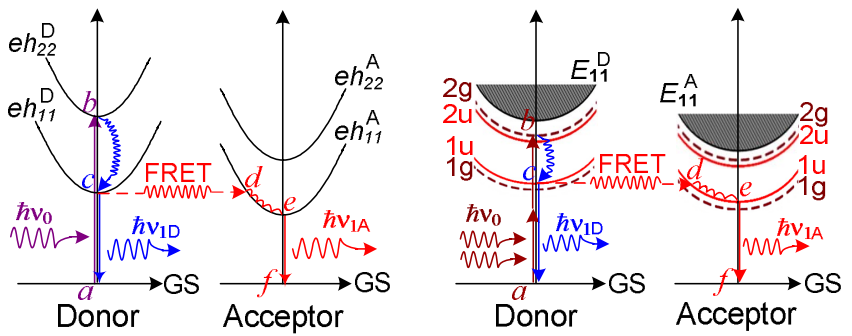

FIG. 5 (color online). (Left) Schematic EET from donor (D) to smaller gap acceptor (A). $(a \rightarrow b)$ exciton absorption at $e h_{22}^{D}$; $(b \rightarrow c)$ fast relaxation to $e h_{11}^{D} ;(c \rightarrow e)$ FRET from $e h_{11}^{D}$ to $e h_{11}^{A}$; $(e \rightarrow f)$ radiative recombination at $e h_{11}^{A}$. (Right) Scheme for two-photon excitation, where $1 \mathrm{~g}, 1 \mathrm{u}, 2 \mathrm{u}, 2 \mathrm{~g}$ are the even $(\mathrm{g})$ and odd (u) exciton states associated with $E_{11}$, adapted from [5].

cific donor and acceptor couples. However, the spectrum in Fig. 3(b) excited at the $(5,4) e h_{11}$, reproduces the profile of the absorption in Fig. 3(c) above $850 \mathrm{~nm}$, with no $(n, m)$ preference. This suggests that the factor ruling EET in bundles is concentration, not symmetry, diameter, or gap difference; thus, exciton tunneling is not the dominant mechanism.

Photon exchange is exciton-photon coupling with no direct donor-acceptor interaction. It has a smaller dependence on donor-acceptor distance $\left(R_{\mathrm{DA}}\right)$ than FRET; thus, it can become significant for much longer distances. However, the lack of significant EET features in isolated tube solutions $[3,17]$, combined with the low quantum efficiency [2], suggest that even if photon exchange might exist between bundles or between isolated SWNTs, it is not dominant between adjacent tubes in a given bundle.

FRET is a very efficient EET mechanism via resonant, near-field, dipole-dipole interaction [11-15]. It is commonly observed in biological systems, conjugated polymers, wires, dots [11-15], where it dominates at short and intermediate distances $(0.5-10 \mathrm{~nm})$ [11-15]. Its efficiency is determined by the spectral overlap of donor emission and acceptor absorption, by $R_{\mathrm{DA}}$, and by the relative orientation of emission and absorption dipoles [11]. The transfer rate is proportional to $R_{\mathrm{DA}}^{-6}$ [11]. The FRET efficiency in bundles is expected to be high. The emissionabsorption overlap between large and small gap tubes depends on the specific donor-acceptor couple. However, the fast EET rate can allow excitons to be cascadedly transferred from donor to acceptor, even when a small emission-absorption overlap is present (Fig. 2), via intermediate gap tubes within a bundle. SWNTs in bundles are parallel, giving a maximum dipole orientation factor. They have small wall-to-wall distance. This makes higher multipolar contributions possible as well [11,12]. Indeed, PL quenching of CdSe-ZnS dots conjugated to SWNTs was reported due to FRET from dots to tubes [15]. This further suggests FRET to be dominant. This process is schematically represented in Figs. 5(a) and 5(b) for both one and two photon measurements.
In summary, we presented a thorough PL investigation of nanotube bundles. We have shown that the apparently complex absorption and emission can be explained by EET between adjacent semiconducting tubes. By studying the spectral evolution for increasing bundle size, we assigned all EET peaks. We argue that Förster interaction between excitons dominates the transfer process. This is highly efficient in bundles, adding a major relaxation channel for excitons, explaining the low luminescence yield of large gap tubes. Thus, contrary to what is usually assumed, bundles could be ideal for high yield optoelectronics. Furthermore, energy transfer fingerprints bundles with different semiconducting tubes concentration, offering a way to monitor the composition of solutions and films, a key need for research and applications.

We acknowledge D. Prezzi, A. Hartschuh, T. Hertel, A. Rubio for useful discussions; funding from EPSRC Grants No. GR/S97613, No. EP/E500935/1, the Ministry of Information and Communication, Republic of Korea (No. A1100-0501-0073), the Royal Society, and the Leverhulme Trust.

*acf26@eng.cam.ac.uk

[1] S. Reich, C. Thomsen, and J. Maultzsch, Carbon Nanotubes (Wiley, Weinheim, 2004).

[2] M. J. O'Connell et al., Science 297, 593 (2002).

[3] S. M. Bachilo et al., Science 298, 2361 (2002).

[4] F. Wang et al., Science 308, 838 (2005).

[5] J. Maultzsch et al., Phys. Rev. B 72, 241402(R) (2005).

[6] V. Perebeinos et al., Phys. Rev. Lett. 92, 257402 (2004).

[7] Y.Z. Ma et al., Phys. Rev. Lett. 94, 157402 (2005).

[8] J. A. Misewich et al., Science 300, 783 (2003).

[9] J. Chen et al., Science 310, 1171 (2005).

[10] O. N. Torrens et al., Nano Lett. 6, 2864 (2006).

[11] T. Förster, Discuss. Faraday Soc. 27, 7 (1959).

[12] C. R. Kagan et al., Phys. Rev. Lett. 76, 1517 (1996).

[13] S. R. Adams et al., Nature (London) 349, 694 (1991).

[14] K. Becker et al., Nat. Mater. 5, 777 (2006).

[15] V. Biju et al., J. Phys. Chem. B 110, 26068 (2006).

[16] B. Kitiyanan et al., Chem. Phys. Lett. 317, 497 (2000).

[17] S. M. Bachilo et al., J. Am. Chem. Soc. 125, 11186 (2003).

[18] V. Perebeinos et al., Phys. Rev. Lett. 94, 027402 (2005).

[19] F. Plentz et al., Phys. Rev. Lett. 95, 247401 (2005).

[20] Y. Miyauchi and S. Maruyama, Phys. Rev. B 74, 035415 (2006).

[21] S. Reich et al., Phys. Rev. B 65, 155411 (2002).

[22] F. Wang et al., Phys. Rev. Lett. 96, 167401 (2006).

[23] See EPAPS Document No. E-PRLTAO-99-034738 for a supplementary figure of corresponding absorption peaks. For more information on EPAPS, see http://www.aip.org/ pubservs/epaps.html.

[24] H. Htoon et al., Phys. Rev. Lett. 94, 127403 (2005).

[25] S. G. Chou et al., Phys. Rev. Lett. 94, 127402 (2005).

[26] C. D. Spataru et al., Phys. Rev. Lett. 92, 077402 (2004).

[27] A. Hagen et al., Phys. Rev. Lett. 95, 197401 (2005).

[28] H. Hirori et al., Phys. Rev. Lett. 97, 257401 (2006).

[29] C. D. Spataru et al., Phys. Rev. Lett. 95, 247402 (2005).

[30] M. G. W. Alexander et al., Phys. Rev. B 41, 12295 (1990). 